\section{The quiescent fraction of chronic myeloid leukemic stem cells depends on BMPR1B, Stat3 and BMP4-niche signals to persist in patients in remission}

\author{
Sandrine Jeanpierre,$^{1,2,3,4,45^{*}}$ Kawtar Arizkane ${ }^{1,2,3,4^{*}}$ Supat Thongjuea, ${ }^{6}$ \\ Elodie Grockowiak, ${ }^{1,2,3,4}$ Kevin Geistlich, ${ }^{1,2,3,4}$ Lea Barral, ${ }^{1,2,3,4}$ \\ Thibault Voeltzel, ${ }^{1,2,3,4}$ Anissa Guillemin, ${ }^{7}$ Sandrine Gonin-Giraud, ${ }^{7}$ \\ Olivier Gandrillon, ${ }^{7}$ Franck-Emmanuel Nicolini, ${ }^{1,2,3,4,5}$ Adam J. Mead, ${ }^{8}$ \\ Véronique Maguer-Satta ${ }^{1,2,3,4 \pi}$ and Sylvain Lefort ${ }^{1,2,3,4 *}$
}

${ }^{1}$ CNRS UMR5286, Centre de Recherche en Cancérologie de Lyon, 69000 Lyon, France; IInserm U1052, Centre de Recherche en Cancérologie de Lyon, 69000 Lyon, France; ${ }^{3}$ Université de Lyon, 69000, Lyon, France; ' Department of Signaling of Tumor Escape, Lyon, France; ${ }^{5}$ Centre Léon Bérard, 69000 Lyon, France; ${ }^{6}$ MRC WIMM Centre for Computational Biology, Weatherall Institute of Molecular Medicine, NIHR Oxford Biomedical Research Centre, John Radcliffe Hospital, Oxford, UK; ' ${ }^{2}$ aboratoire de Biologie et Modélisation de la Cellule, LBMC - Ecole Normale Supérieure - Lyon, Université Claude Bernard Lyon - Centre National de la Recherche Scientifique: UMR5239 - Institut National de la Santé et de la Recherche Médicale: U1210 - Ecole Normale Supérieure de Lyon, 69007 Lyon, France and ${ }^{8} \mathrm{Haemopoietic} \mathrm{Stem} \mathrm{Cell} \mathrm{Biology} \mathrm{Laboratory,} \mathrm{Weatherall} \mathrm{Institute}$ of Molecular Medicine, University of Oxford, Oxford, UK

*SJ and KA contributed equally as co-first author.

"VM-S and SL contibuted equaly as co-senior authors.

\section{ABSTRACT}

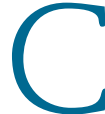
hronic myeloid leukemia arises from the transformation of hematopoietic stem cells by the BCR-ABL oncogene. Though transformed cells are predominantly BCR-ABL-dependent and sensitive to tyrosine kinase inhibitor treatment, some BMPR1B ${ }^{+}$leukemic stem cells are treatment-insensitive and rely, among others, on the bone morphogenetic protein (BMP) pathway for their survival via a BMP4 autocrine loop. Here, we further studied the involvement of BMP signaling in favoring residual leukemic stem cell persistence in the BM of patients having achieved remission under treatment. We demonstrate by single-cell RNASequencing analysis that a sub-fraction of surviving BMPR1B+ leukemic stem cells are co-enriched in BMP signaling, quiescence and stem cell signatures, without modulation of the canonical BMP target genes, but enrichment in actors of the Jak2/Stat3 signaling pathway. Indeed, based on a new model of persisting $\mathrm{CD} 34^{+} \mathrm{CD} 38^{-}$leukemic stem cells, we show that BMPR1B $^{+}$cells display co-activated Smad1/5/8 and Stat3 pathways. Interestingly, we reveal that only the $\mathrm{BMPR}^{+} \mathrm{B}^{+}$cells adhering to stromal cells display a quiescent status. Surprisingly, this quiescence is induced by treatment, while non-adherent $\mathrm{BMPR} 1 \mathrm{~B}^{+}$cells treated with tyrosine kinase inhibitors continued to proliferate. The subsequent targeting of BMPR1B and Jak2 pathways decreased quiescent leukemic stem cells by promoting their cell cycle re-entry and differentiation. Moreover, while Jak2-inhibitors alone increased BMP4 production by mesenchymal cells, the addition of the newly described BMPR1B inhibitor (E6201) impaired BMP4-mediated production by stromal cells. Altogether, our data demonstrate that targeting both BMPR1B and Jak2/Stat3 efficiently impacts persisting and dormant leukemic stem cells hidden in their BM microenvironment.

\section{Introduction}

Chronic myeloid leukemia (CML) represents a unique model of neoplasia driven via stem cell (SC) transformation, which is triggered by the BCR-ABL oncogene. Without treatment, this disease progresses to an inexorable fatal blast crisis. Apart

\section{Ferrata Storti Foundation}

\section{Correspondence:}

VÉRONIQUE MAGUER-SATTA

veronique.maguer-satta@lyon.unicancer.fr

SYLVAIN LEFORT

sylvain.lefort@lyon.unicancer.fr

Received: July 22, 2019

Accepted: January 27, 2020.

Pre-published: January 30, 2020.

https://doi.org/10.3324/haematol.2019.232793

(C2021 Ferrata Storti Foundation

Material published in Haematologica is covered by copyright. All rights are reserved to the Ferrata Storti Foundation. Use of published material is allowed under the following terms and conditions:

https://creativecommons.org/licenses/by-nc/4.0/legalcode. Copies of published material are allowed for personal or internal use. Sharing published material for non-commercial purposes is subject to the following conditions:

https://creativecommons. org/licenses/by-nc/4.0/legalcode, sect. 3. Reproducing and sharing published material for commercial purposes is not allowed without permission in writing from the publisher. 
from allogeneic stem cell transplantation, until 2001, interferon- $\alpha(\mathrm{IFN}-\alpha)$ was extensively used for the treatment of chronic phase (CP) CML and induced stable remission in some patients. ${ }^{1}$ This was correlated with long-term survival, ${ }^{2}$ but was also responsible for numerous side effects leading to drug dose reduction and lack of response. Tyrosine kinase inhibitors (TKI), such as imatinib (IM), are targeted therapies that specifically inhibit BCR-ABL tyrosine kinase activity. TKI revolutionized the management of CML patients and currently represent the first-line treatment.

However, TKI fail to eliminate leukemic stem cells (LSC), which persist in patients displaying complete cytogenetic remission (CCyR), ${ }^{3,4}$ as evidenced by the indefinite detection of residual $\mathrm{BCR}-\mathrm{ABL}^{+}$cells in their blood. These cells are most likely responsible for the high rate of relapse after treatment discontinuation, even in patients with a durable undetectable status. ${ }^{5}$ Indeed, LSC are highly heterogeneous, and single-cell transcriptomic analyses linked specific subpopulations to a primitive quiescent phenotype that persists throughout therapy, conferring cells upon treatment cessation with the ability to proliferate extensively, regenerate or acquire resistance. ${ }^{6,7}$

As LSC are insensitive to TKI, this strongly suggests that their survival is independent of BCR-ABL activity, relying on other factors, including interactions with the bone marrow (BM) microenvironment. ${ }^{8-12}$ Among the cytokines produced by the BM stroma, bone morphogenetic proteins (BMP) are a group of growth factors involved in many processes, including stem cell regulation. During embryogenesis, BMP4 is crucial for mesodermal cell commitment to the hematopoietic lineage. ${ }^{13}$ BMP4-deficient mice display a reduced number of hematopoietic stem cells (HSC), while the engraftment of wild-type HSC into BMP4-deficient mice impairs the repopulating activity of these cells. ${ }^{14}$ Additionally, BMP4 regulates the maintenance of human primitive cord blood progenitors. ${ }^{15}$ In adults, BMP regulate distinct characteristics of HSC and parameters of their niche. ${ }^{16}$ For example, BMP4 induces $\mathrm{CD} 34^{+}$progenitor differentiation into megakaryocytes ${ }^{17}$ whereas BMP2 favors erythropoietic commitment. ${ }^{18}$ The BMP pathway that co-regulates the fate and proliferation of HSC and interactions with their niche has been reported in several types of leukemia, ${ }^{19}$ such as acute myloid leukemia (AML), ${ }^{20,21}$ and is dysregulated in CML.22-24 Indeed, CP-diagnosed CML patients show higher concentrations of soluble BMP2 and BMP4 in their $\mathrm{BM}$ compared to normal BM donors. Furthermore, the BMP receptor type-1B (BMPR1B) is over-expressed in CP-diagnosed CD34+ CML cells. Soluble BMP, secreted into the tumor niche maintain a fraction of BMPR1B+ LSC and amplify leukemic progenitors, contributing to disease progression. ${ }^{22}$ These alterations sustain a permanent pool of LSC and progenitors expressing high levels of BMPR1B receptor, which evolve upon treatment $t^{25}$ and progressively implement a BMP4 autocrine loop, leading to TKI-resistance. ${ }^{26,27}$

Having unraveled a majority of the interactions/mechanisms underlying the BMP-associated survival of $\mathrm{BMPR}^{+} \mathrm{B}^{+} \mathrm{LSC}$ in the BM niche, we herein further investigated the spatio-temporal setting of persistence mechanisms in CML TKI-responsive patients after remission. It remains a major therapeutic challenge to achieve complete recovery through eradication of $B M P R 1 B^{+} L S C$. We identify BMP4/BMPR1B as a major bi-directional signal driving microenvironment-dependent LSC persistence alongside the Jak2-Stat3 pathway. Our data further show that targeting both BMP and Jak2/Stat3 signaling efficiently promotes LSC cell cycle re-entry and differentiation, enabling their efficient eradication.

\section{Methods}

\section{Cells}

Bone marrow samples were obtained either from healthy BM donors for allogeneic transplant or from peripheral blood (PB), or from the BM of CML patients diagnosed to be in $\mathrm{CP}$ prior to or post TKI treatment (from patients achieving CCyR). All donors provided written informed consent in accordance with the Declaration of Helsinki. Studies were approved by local ethics committee bylaws. Definitions of response respected the last European LeukemiaNet Recommendations. ${ }^{28}$ Mesenchymal stem cells (MSC) were isolated from BM samples and cultured as described. ${ }^{26,29}$ TF1 cell lines were obtained and authenticated as described. ${ }^{22,30}$ TF1-BA, TF1-BAP cells were cultured in RPMI1640 containing $10 \%$ fetal calf serum (FCS), with or without $2 \mu \mathrm{M} \mathrm{IM}$. HS5 and HS27A cell lines were obtained from ATCC and cultured in DMEM and RPMI1640 containing 10\% FCS.

\section{RNA analysis}

The quantitative reverse transcription (RT) polymerase chain reaction (PCR) protocol, reagents, and primers are described by Laperrousaz et al. ${ }^{22}$ Single-cell RNA sequencing and gene set enrichment analyses were performed as previously described.? The BMP signature was designed as presented in the Online Supplementary Table S1. Briefly, we took 245 cells from groups A and $B$ derived from 16 patients, of whom 11 and 5 were good and poor responders, respectively. ${ }^{7}$ All cells analyzed in single-cell RNA sequencing were BCR-ABL ${ }^{+}$cells as verified by qualitative (q)PCR analysis. High-throughput microfluidic-based RT-qPCR was performed following the Fluidigm protocols (PN 68000088 K1) as described. ${ }^{31}$

\section{Flow cytometry}

Extracellular staining of cells was performed using PECy5-conjugated anti-CD34, APC-conjugated anti-CD38, PE-conjugated anti-CD71 (BD Biosciences) or PE-conjugated anti-GPA (Invitrogen). For intracellular staining, cells were fixed with $4 \%$ paraformaldehyde, and washed and permeabilized with $80 \%$ ethanol before adding PE-conjugated anti-P-Smad1/5/8 (Cell Signaling) or AF647-conjugated anti-P-Stat3 (Biolegend), or PE-conjugated anti-Ki67 (BD Biosciences).

\section{Bone morphogenetic protein quantification}

Bone marrow plasma or HS5/HS27A supernatants were precleared of cellular debris by rapid centrifugation and processed for BMP4 ELISA quantification (R\&D Systems) as previously described. ${ }^{22}$

\section{Western blotting}

Western blot was performed using TF1-BA and TF1-BAP cells treated with IM $(2 \mu \mathrm{M})$, AG490 $(25 \mu \mathrm{M})$ or E6201 (100 nM) during 24 hours (h), proteins were then extracted using RIPA buffer and $40 \mu \mathrm{g}$ of proteins were loaded. Membranes were incubated with monoclonal-antibodies against P-ERK (\#4370), ERK (\#4695), P-STAT3 (\#9145), STAT3 (\#9139), Caspase 3 (\#9662). P-ABL (\#2865), P-CRKL (\#3181), P-Smad1/5/8 (\#13820), GAPDH (\#8884) (Cell Signalling Technology), BCR-ABL (ThermoFisher, \#MA1153) and Smad (SantaCruz Technology, \#sc6031). ${ }^{21}$ 


\section{Functional assays}

Colony forming cell (CFC) and long-term culture-initiating cell (LTC-IC) assays were performed as reported. ${ }^{22}$

\section{Chemical inhibitors}

Cells were treated with AG490 (Sigma-Aldrich) and/or E6201 (Spirita Oncology, LLC), a BMPR1B inhibitor, also shown to inhibit MEK1/2 and FLT3 without affecting BMPR1A or JAK2 ${ }^{32-34}$ (Online Supplementary Table S2).

\section{Statistical analysis}

Mean comparisons were performed using the bilateral MannWhitney test (paired or unpaired as required) and correlations were determined with the Pearson bilateral test, using the GaphPadPrism software (San Diego, CA, USA). ${ }^{*} P \leq 0.05,{ }^{* *} P \leq 0.01$, ${ }^{* * *} P \leq 0.001$, were considered statistically significant.

\section{Results}

At remission, persistent chronic myeloid leukemia cells are enriched in bone morphogenetic protein and quiescence signaling pathways

Recent single-cell analyses revealed the heterogeneity of the SC compartment in the BM of CML patients with distinct sub-fractions of LSC that displayed different molecular signatures. ${ }^{7}$ This study demonstrated the selective persistence of a BCR-ABL SC subset (group A) already present at diagnosis and from which leukemic cells emerge at relapse upon disease progression. This LSC sub-fraction progressively accumulates at the expense of another LSC sub-fraction (group B) in patients treated with TKI. Interestingly, in contrast to group $B$, $\mathrm{BCR}-\mathrm{ABL}^{+}$cells from group $\mathrm{A}$ are highly quiescent. At the beginning of the study reported here, we hypothesized that BMP signaling could be involved in the maintenance of group A LSC in CML patients that achieved remission, due to the involvement of this pathway in TKI resistance. To address this and gain further insight into mechanisms underlying LSC persistence, we analyzed RNAsequencing (RNASeq) data obtained from Giustacchini et al. for BMP pathway actors. Compared to the highly proliferative group $B$ that disappeared upon TKI treatment, cells from group A displayed an enhanced BMP signaling pathway (Figure $1 \mathrm{~A}$ and Online Supplementary Table S1). Both persistent LSC from group $A$ and $B$ expressed BMPR1B transcripts at variable levels (Figure 1B, left panel). However, unlike our previous observations in resistant $\mathrm{LSC}^{26}$ persistent LSC were devoid of autocrine BMP4 production, suggesting their dependence upon BMP4-producing cells of their niche (Figure 1B, right panel). Interestingly, neither group exhibited a significant difference in the comparative level of classical BMP-target genes, as illustrated in Online Supplementary Figure S1A for ID1, ID2, RUNX1 and $R U N X 2$ gene expression. In addition, single cell analyses of $\mathrm{BMPR}_{1 \mathrm{~B}}{ }^{+}$cells from each group revealed that LSC of group A scarcely expressed Id1 or Runx1 (8\% of group A cells) (Online Supplementary Figure $S 1 B$, left panel) unlike cells of group B that frequently ( $50 \%$ of group B cells) coexpressed these genes with BMPR1B (Online Supplementary Figure $S 1 B$, right panel).

Taken together, our data indicate that the selective persistence of a quiescent $\mathrm{BCR}-\mathrm{ABL}^{+} \mathrm{LSC}$ subset (group A) upon treatment is correlated in these cells with a signifi- cant enrichment in the BMP signaling molecular signature, unrelated to the expression of BMP target genes.

Persisting quiescent leukemic stem cells are characterized by bone morphogenetic protein and Stat3 co-activation

We then investigated whether $\mathrm{BMPR} \mathrm{B}^{+}$cells could activate alternative non-canonical pathways in LSC of groups $\mathrm{A}$ and $\mathrm{B}$ to enable them to persist in the $\mathrm{BM}$ microenvironment despite TK treatment. Based on these single cell RNAseq data, we identified a specific correlation between BMPR1B and STAT3 pathways exclusively in group A (quiescent cells), though this was not correlated with the expression of IL6ST (gp130) (Figure 1C, left panel). Conversely, BMPR1B expression was associated with kinases like Tyk2 and MAP3K8 in the proliferative group $\mathrm{B}$ (Figure 1C, right panel). No correlation between $B M P R 1 B$ and Stat5 signaling was identified in either group (Online Supplementary Figure S1C).

We next evaluated the functional link between these two pathways. However, it was not technically possible to access viable $\mathrm{BMPR}^{+} \mathrm{B}^{+}$quiescent LSC subpopulation by cell sorting BM cells from patients in remission due to their extremely low frequency and the fact that it was impossible to distinguish BCR-ABL cells from dominant non-BCR-ABL stem cells. Therefore, owing to the scarcity of the sorted BMPR1B+-LSC sub-fraction from the BM of CML patients in remission, we developed a novel CML model of TKI-persistent immature cells. We used an established TF1-BCR-ABL (TF1-BA) cell line ${ }^{22}$ selected to persist upon chronic imatinib-IM (2 $\mu \mathrm{M}$ of TKI IM) exposure, designated as TF1-BAP (TF1-BCR-ABL persistent) (Figure 2A). Persistence of TF1-BAP cells was accompanied by a slightly higher level of BMPR1B as observed by immunfluorescence, western blot and qPCR (Figure 2B and C and Online Supplementary Figure S2A). Similarly to RNAseq data from primary BM cells of patients in remission (Figure 1B), TF1-BAP cells do not implement an autocrine BMP4 production, but inversely seemed to repress its production (Online Supplementary Figure S2B), corroborating our previous report in $\mathrm{CD} 34^{+} \mathrm{CML}$ cells from patients that achieved CCyR. ${ }^{26}$

The TF1 human cell line displayed immature properties similar to human hematopoietic stem and progenitor cells, including the immature $\mathrm{CD}_{34}{ }^{+} \mathrm{CD} 38^{-}$(stem) and $\mathrm{CD} 34^{+} \mathrm{CD}_{38}{ }^{+}$(progenitor) phenotypes, and their ability to generate colony-forming progenitor cells (CFC) in a semi-solid medium (see Figure $2 \mathrm{~A}$ and Online Supplementary Figure S3A) from both erythroid and myeloid lineages. ${ }^{35}$ Chronic treatment of TF1-BA cells with TKI led to a severe reduction in the $\mathrm{CD} 34^{+} \mathrm{CD} 38^{+}$ sub-fraction in ensuing TF1-BAP cells, which were thus characterized by a CD34+CD38- phenotype (Figure 2D). We then evaluated the CFC output (Figure 2E and Online Supplementary Figure S3A) or LTC-IC potential (Figure 2F and Online Supplementary Figure $S 3 B$ ) following TKI persistence. We observed a drastic decrease in the ability of TF1-BAP cells to form CFC compared to TF1-BA (Figure $2 \mathrm{E})$, suggesting a loss of progenitor activity. Moreover, TF1-BAP cells display increased LTC-IC activity (Figure $2 \mathrm{~F}$ ) according to a higher $\mathrm{CD} 34^{+} \mathrm{CD} 38^{-}$phenotype (Figure 2D). However, the W5-CFC colonies, representative of BCR-ABL TF1 immature cells derived LTC-IC, were difficult to observe, as we had frequently experienced with primary CML cells. We also detected a higher expression 
level of stemness-associated transcription factors (NANOG, MYC and FoxO1 and FoxO3a) ${ }^{36-38}$ as well as an increase in genes involved in LSC survival (CD123, TPOR, HIF2a, ALOX5, TWIST-1, BCL2 and BCL-XL) ${ }^{8,36,39-}$ ${ }^{41}$ in TF1-BAP compared to TF1-BA cells (Online Supplementary Figure S2A). Taken together, these data confirmed the leukemic stem-like status of TF1-BAP cells. We also show that, despite increased BCR-ABL protein levels, TF1-BAP display similar activation of P-CRKL (Figure $2 \mathrm{G}$ ), and TKI equally impairs P-BCR-ABL in TF1-BA and TF1-BAP cells (while having no effect on P-Smad1/5/8) (Online Supplementary Figure S4A), indicating that there is no major involvement of the BCR-ABL kinase activity in the persistent phenotype. Therefore, this set of data further sustains that previously described in primary CML-LSC. ${ }^{42}$ Lastly, we used intracellular flow cytometry to measure the level of activation of BMP and Stat3 signaling, and revealed an increase in $\mathrm{P}-\mathrm{Smad1/5/8}$ and double positive P-Smad1/5/8-P-Stat3 levels in TF1BAP cells compared to IM-sensitive TF1-BA cells (Figure $2 \mathrm{H})$. However, no difference was observed in total PStat3 levels between the TF1-BA and TF1-BAP, as evidenced from western blot and flow cytometry analyses (Figure $2 \mathrm{H}$ and Online Supplementary Figure S4B), suggesting that in TKI persistent cells a specific co-activation of Smad/Stat3 occurs.

Altogether these data indicate that the TF1-BAP cell line is a representative model of the primary CML persistent LSC group A characterized by a $\mathrm{BMPR}_{1} \mathrm{~B}^{+}$expression, stem-like status, the absence of autonomous BMP4 production, and the co-activation of P-Smad1/5/8-P-Stat3 signals.
A

BMP Signaling pathway

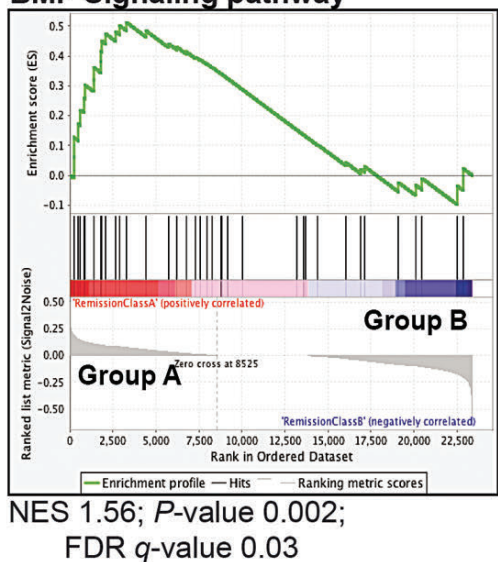

FDR $q$-value 0.03
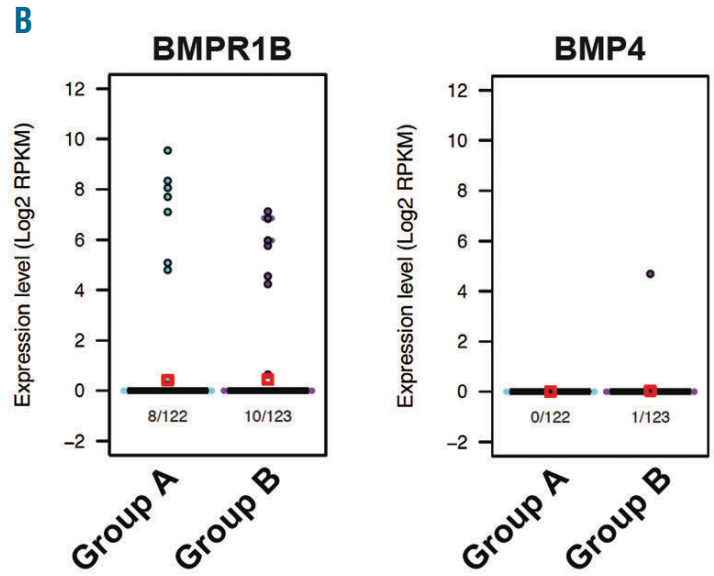
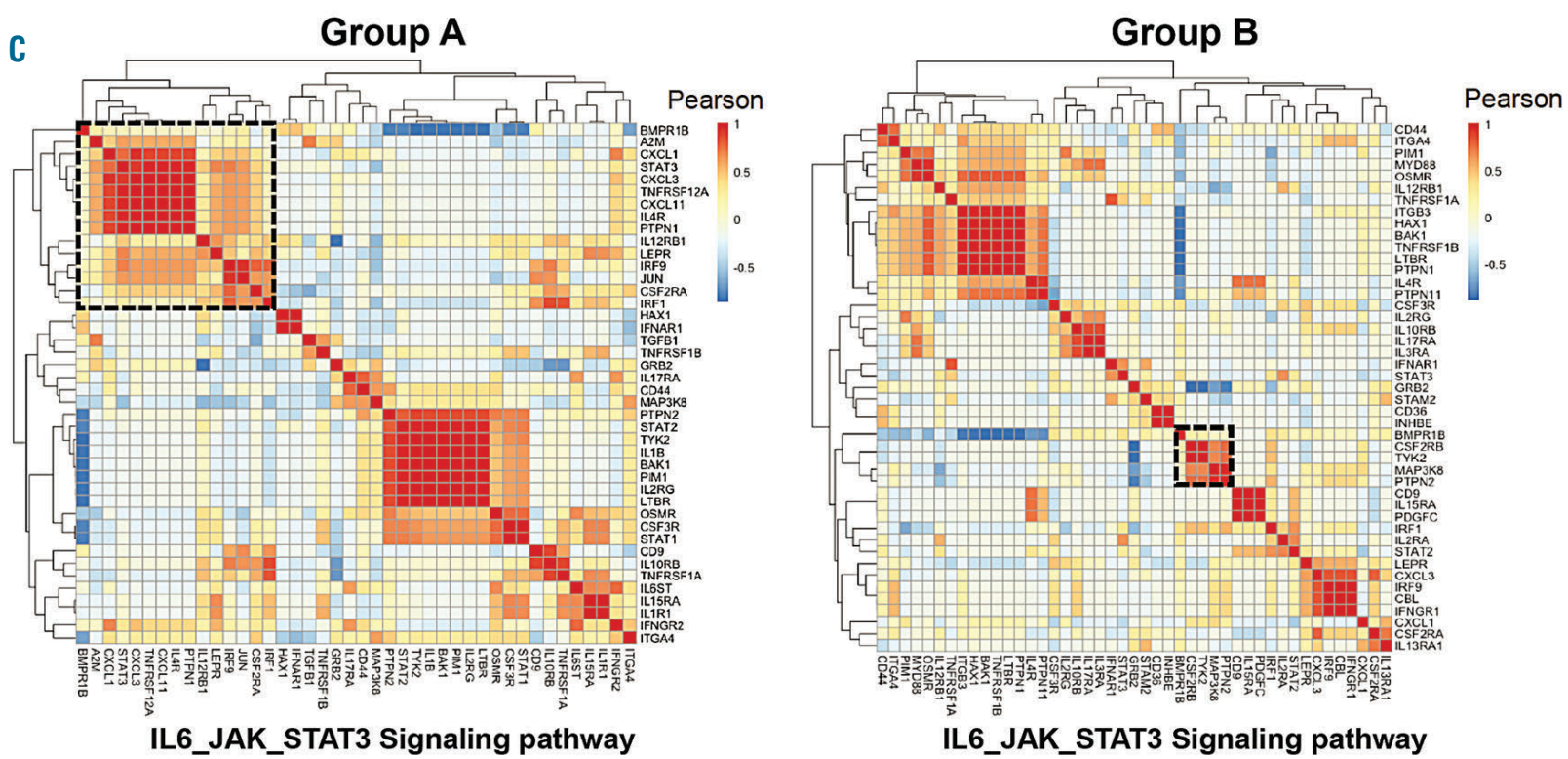

Figure 1. BMPR1B-positive chronic myeloid leukemia (CML) persistent cells signal through the Stat3 pathway. (A) Gene set enrichment analysis (GSEA) of group A versus group $B B C R-A B L^{+}$stem cells $(S C)$ at remission ( $n=122$ and $n=123$, respectively). Gene set shown is bone morphogenetic proteins (BMP) signaling pathway. (B) Beeswarm plot of BMPR1B (left) or BMP4 (right) expression between group A (light blue; $\mathrm{n=122}$ ) and group $\mathrm{B} \mathrm{BCR}^{-\mathrm{ABL}} \mathrm{SC}$ from patients at remission (purple; $n=123$ ). Number of cells analyzed and displaying amplification for the selected genes are shown below the plot. Average gene expression level is indicated by red squares; boxes represent the median and quartiles of gene expression levels. (C) Heat map shows hierarchical clustering of IL6_JAK_STAT3 signaling pathway genes in BMPR1B ${ }^{+}$cells $(n=8)$ from group A BCR-ABL $S C$ (left) or group B BCR-ABL $S C$ (right). FDR: false discovery rate. 
BMP4/BMPR1B contribute to maintaining leukemic cells in a non-differentiated state by regulating Stat3 signaling

Next, we evaluated the importance of BMP and Stat3 signaling in protecting $\mathrm{BCR}-\mathrm{ABL}^{+}$cells from TKI-induced cell death. We used the synthetic small molecule inhibitor E6201, which has a strong affinity for BMPR1B (Online Supplementary Table S2). Having shown the relevance of the TF1-BAP persistent CML cell model, with increased BMPR1B expression and P-Smad1/5/8-P-Stat3 activity, we also evaluated the impact of a Jak2 inhibitor (AG490) used alone or in combination with E6201. We confirmed in TF1-BAP that E6201 inhibited P-Smad1/5/8 (Figure 3A), while, as expected, AG490 decreased P-Stat3 (Online Supplementary Figure S5A). Neither E6201 nor AG490 affected the kinase activity of BCR-ABL as revealed by the absence of a significant effect on the main BCR-ABL and ERK targets, P-Ckrl and P-ERK, respectively (Online Supplementary Figure S5B). In addition, no significant change in apoptosis was observed by monitoring caspase3 cleavage (Online Supplementary Figure S5C). Interestingly, E6201 alone also significantly decreased P-Stat3 levels
A

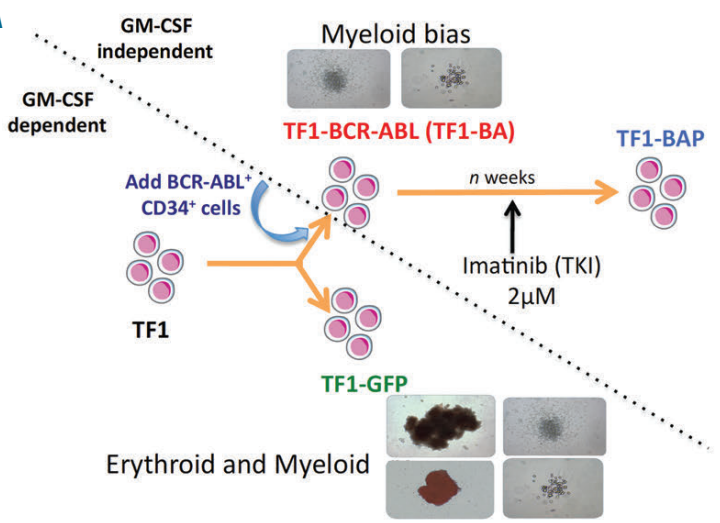

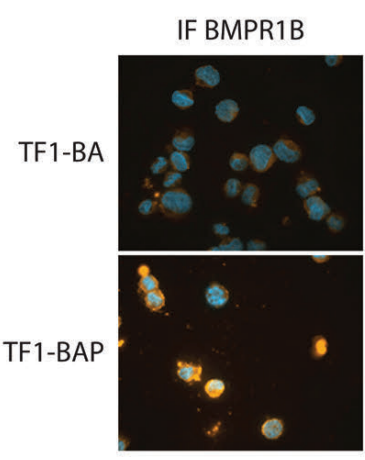

C
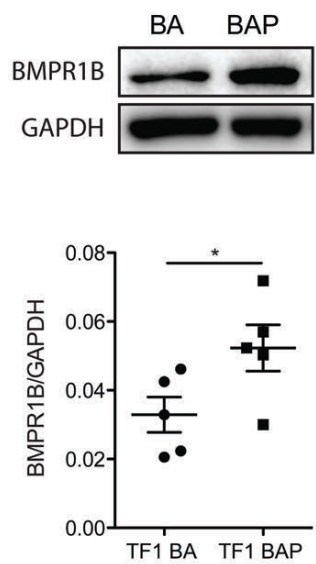

D

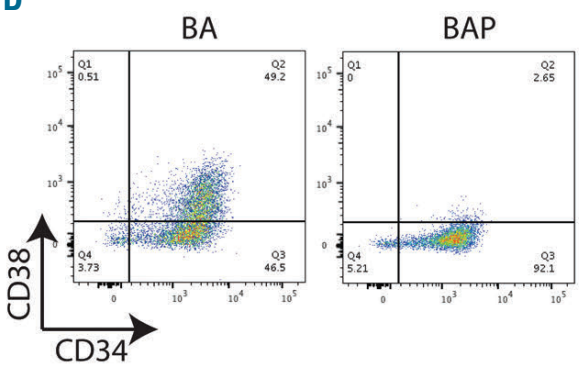

E

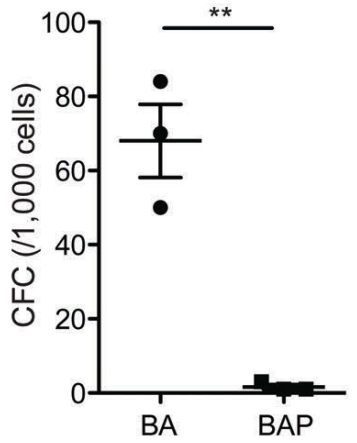

$\mathrm{F}$

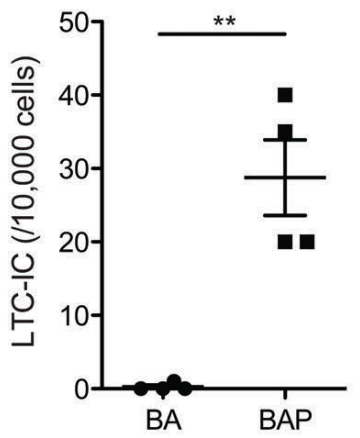

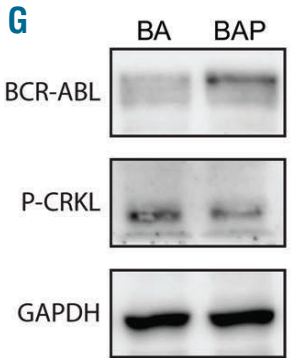

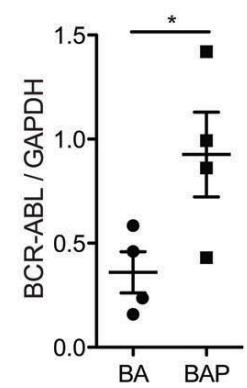

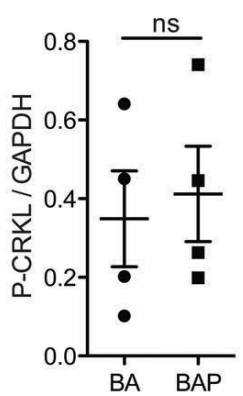
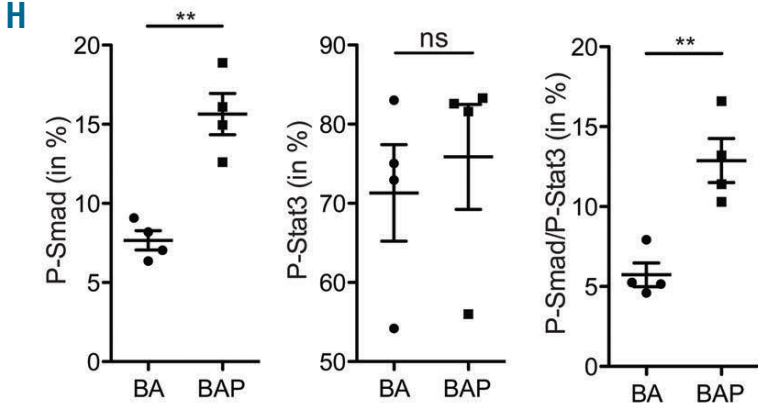

Figure 2. The TF1-BAP model displays similar features as chronic myeloid leukemia (CML) persistent cells. (A) Strategy for generating TF1-BA and TF1-BAP cell lines. (B) Representative images of BMPR1B staining of TF1-BA and TF1-BAP cells. (C) Western blot analysis showing BMPR1B levels in TF1-BA and TF1-BAP cells; Scatter plot showing BMPR1B (GAPDH used as an internal control) $n=5$. (D) Representative FACS plots of TF1-BA and TF1-BAP cells analyzed for their CD34 and CD38 content. (E) Dot plot showing colony forming cell (CFC) activity from TF1-BA and TF1-BAP cells. (F) Dot plot showing long-term culture-initiating cell (LTC-IC) activity from TF1-BA and TF1-BAP cells. (G) Western blot analysis showing BCR-Abl and P-CRKL levels in TF1-BA and TF1-BAP cells. Bar graph showing BCR-Abl and P-CRKL (GAPDH used as an internal control); $n=4$. (H) TF1-BA and TF1-BAP cells were analyzed for their content in P-Smad1/5/8 and P-Stat3. Dot plots represent percentage of PSmad1/5/8 (left panel), P-Stat3 (middle panel) or double positive P-Smad1/5/8-P-Stat3 (right panel). (E-H) Unpaired $t$-test significant: * $P \leq 0.05, * * P \leq 0.01$. ns: not significant. 
A

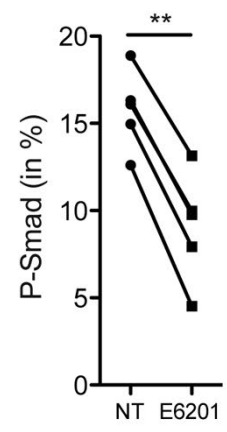

B

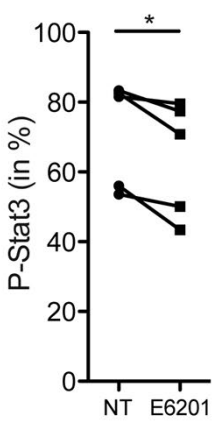

C

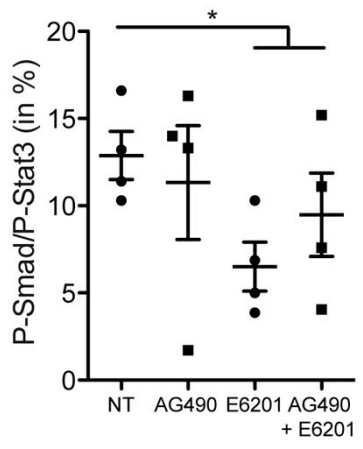

D

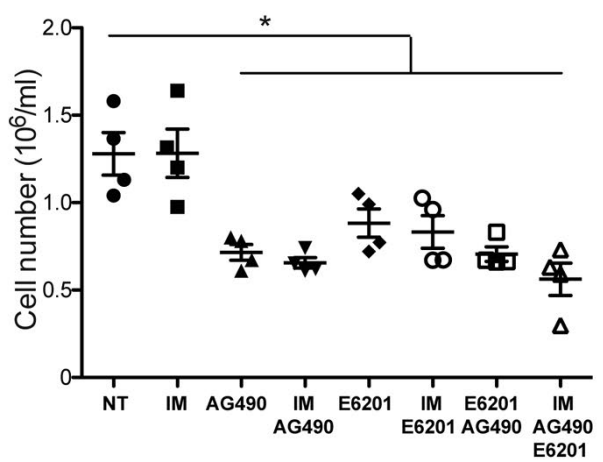

E

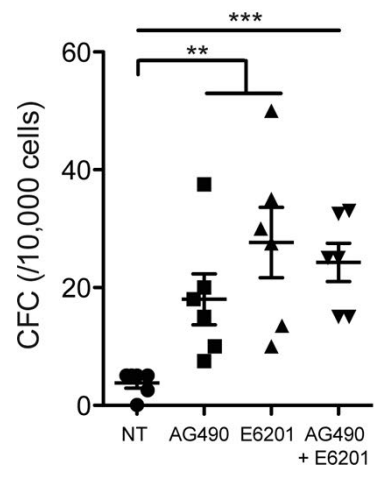

F
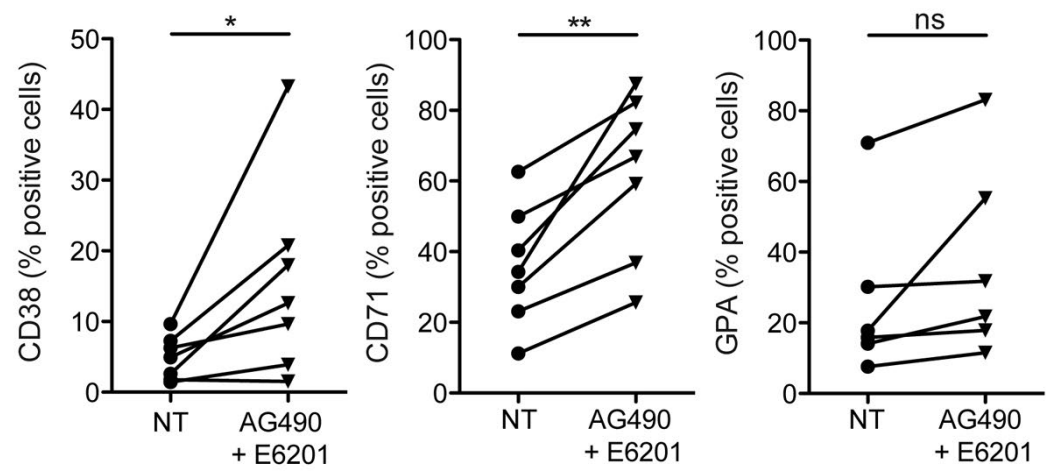

Figure 3. Dual targeting of BMPR1B and Stat3 impairs proliferation and promotes differentiation of chronic myeloid leukemia (CML) persistent cells. (A and B) TF1-BAP cells treated with E6201 (100 nM) for 24 hours $(\mathrm{h})$ and analyzed for their content of P-Smad1/5/8 (A) or P-Stat3 (B). (C) TF1-BAP cells treated or not (NT) with AG490 $(25 \mu \mathrm{M})$ or E6201 (100 nM) for $24 \mathrm{~h}$ and analyzed for their content of double positive P-Smad1/5/8-P-Stat3. (D-F) TF1-BAP cells were treated for 3 days with imatinib (IM) $(2 \mu \mathrm{M})$, and/or AG490 $(25 \mu \mathrm{M})$, and/or E6201 (100 nM), and then (D) cell viability was determined by counting viable cells (Trypan blue exclusion), (E) colony forming cell (CFC) activity was assessed, or (F) cells were analyzed for their content of CD38 (left panel), CD71 (middle panel) or GPA (right panel). Unpaired (C-E) or paired (A and B, F) $t$-test: ${ }^{*} P \leq 0.05, * * P \leq 0.01, * * * P \leq 0.001$. NT: not treated.

(Figure 3B). Moreover, unlike AG490 (that had no impact on the level of P-Smad1/5/8-P-Stat3 signal detected by flow cytometry), E6201 alone or in combination with AG490 decreased the cell subset presenting co-activation of P-Smad1/5/8 and P-Stat3 (Figure 3C and Online Supplementary Figure S5D).

At the functional level, we observed that either AG490 or E6201 or their combination were sufficient to decrease the proliferation of persistent TF1-BAP cells (Figure 3D). The effect of both inhibitors was not modulated by the presence or absence of TKI, which, as expected, by itself had no effect on TF1-BAP cell proliferation (Figure 3D). Treatment of TF1-BAP with AG490 or E6201, alone or in combination, significantly increased the progenitor outcome as evidenced by CFC assays (Figure 3E). We then used flow cytometry to measure the appearance of cell surface markers indicative of early progenitor expansion (CD38 and CD71) and differentiation (GPA) into a nonmyeloid lineage to evaluate whether this increase in CFC and proliferation arrest could be due to the engagement of the pluripotent immature leukemic cell towards a more differentiated stage. Results indicate that inhibition of the JAK2/Stat3 and BMP signaling induced TF1-BAP cells to differentiate into a more committed progenitor status, as indicated by the increased number of $\mathrm{CD}^{3} 8^{+}$(3.26-fold) or CD71 ${ }^{+}$cells $(1.72$-fold) and a trend to increase GPA cell membrane expression (1.42-fold) (Figure 3F).

Collectively, these results suggest that simultaneous inhibition of BMP and JAK2 signaling could induce a decrease in persistent LSC through the induction of their differentiation towards progenitors.

\section{Targeting of BMP4/BMPR1B impairs chronic myeloid leukemia persistent cells mediated by their niche}

As we have previously shown that BMP2 and BMP4 secreted by the niche are drivers of LSC survival and expansion at diagnosis and following the development of resistance, ${ }^{22,26}$ we analyzed whether patients having achieved CCyR displayed normal levels of these molecules following treatment. The level of both BMP2 and BMP4 was significantly reduced in CCyR patients compared to values obtained at diagnosis (Online Supplementary Figure S6A and B). However, a higher (2.7fold) concentration of BMP2 and BMP4 persists in the BM plasma of patients in remission compared to healthy donors (Figure 4A), indicating that, despite eradication of a majority of leukemic cells, soluble BMP remain abnormally elevated in the BM niche. Our former findings also revealed that BMP4, which contributes to LSC survival under TKI treatment, is provided by the leukemic niche. ${ }^{22,26}$ Consequently, we evaluated whether combinations of BMP and/or Jak2 inhibitors (E6201 and AG490, respectively) impacted its production by BM mesenchymal stem cells (MSC). Surprisingly, AG490 alone increased BMP4 expression in both CML (Figure 4B) and healthy MSC (Online Supplementary Figure S6C), while TKI (IM) 
A

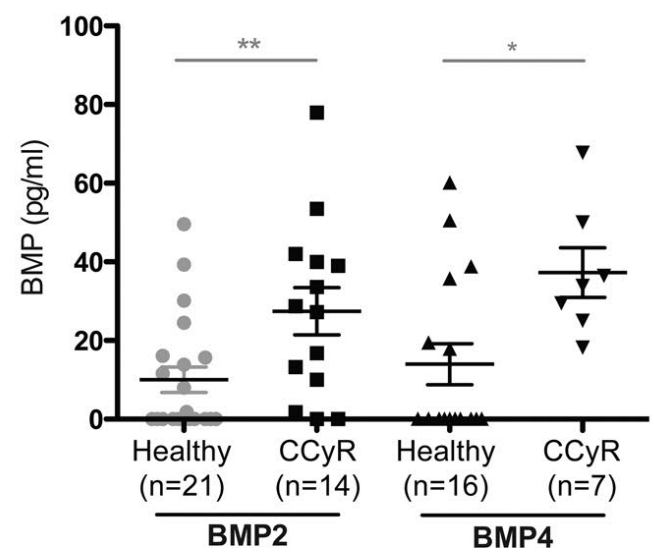

B

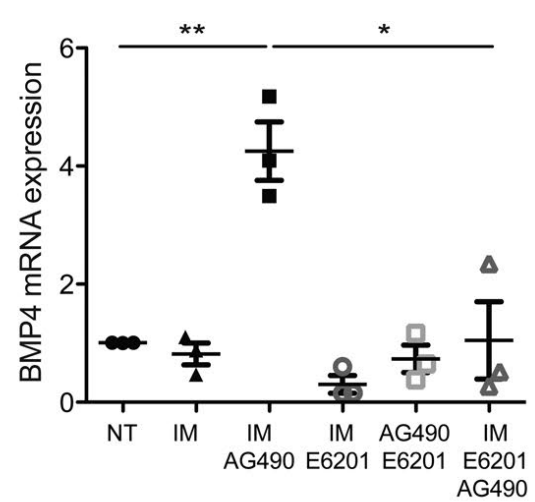

C

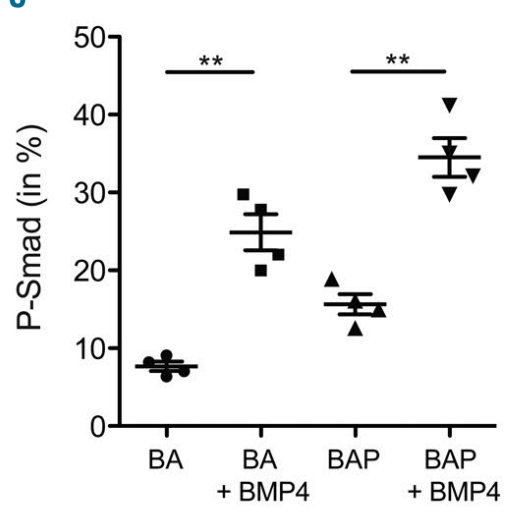

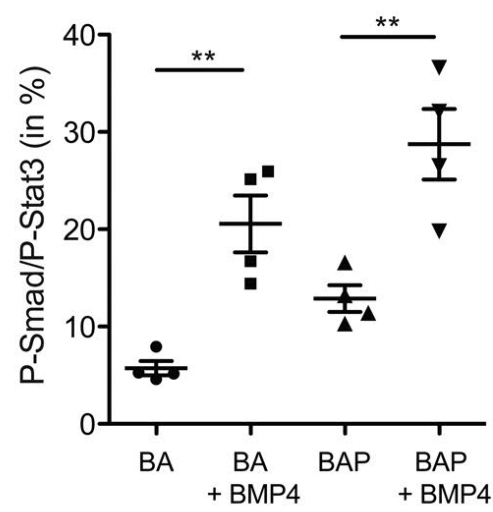

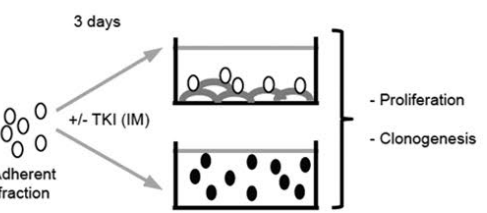

F

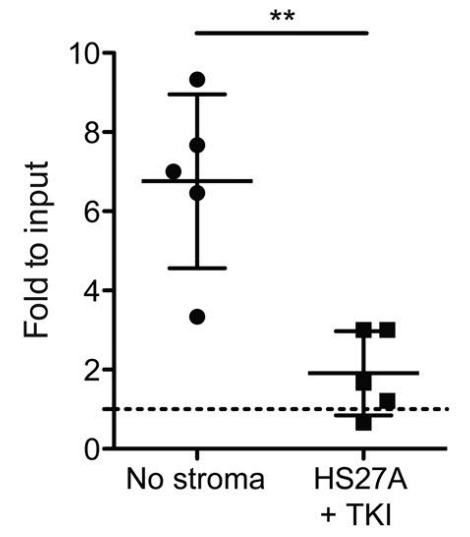

Day 6

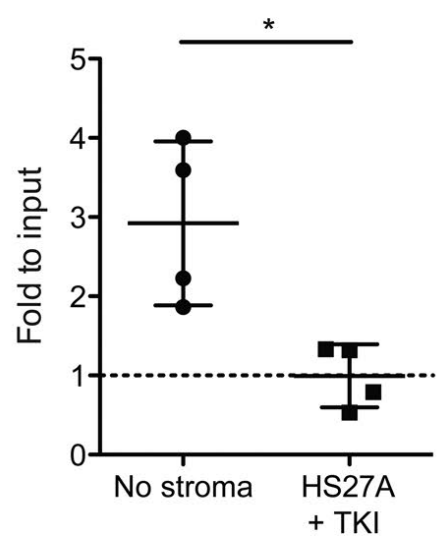

Day 9
D
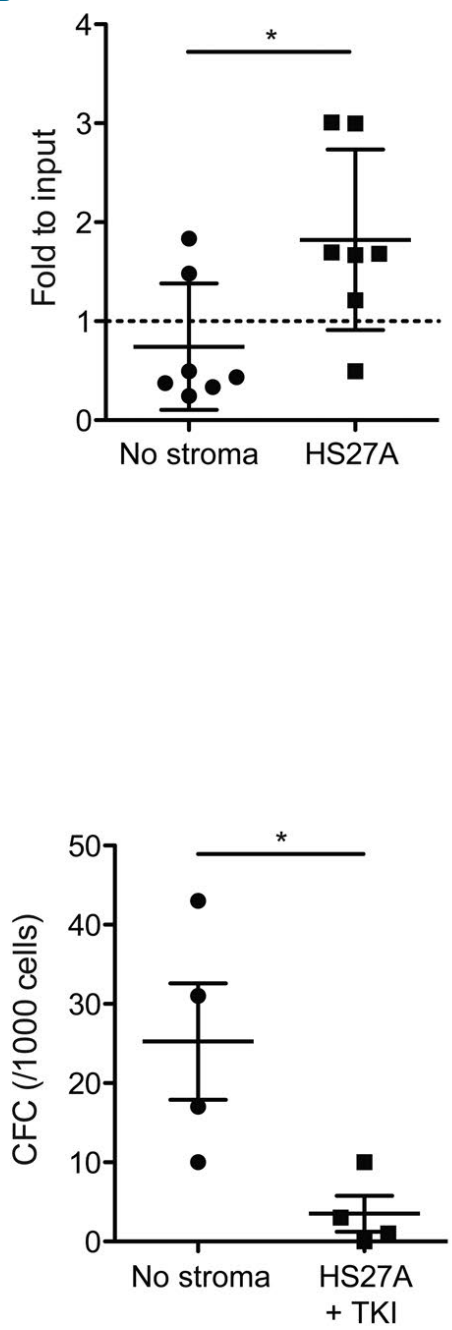

Day 9

Figure 4. Chronic myeloid leukemia (CML) niche promotes tyrosine kinase inhibitor (TKI) persistence of leukemic cells. (A) ELISA quantification of BMP2 and BMP4 in bone marrow (BM) plasma obtained from healthy donors and complete cytogenetic remission (CcyR) patients. Results from individual samples are expressed in picograms per milliliter, and horizontal lines represent mean values \pm standard error of mean (SEM) of the indicated number of analyzed samples. (B) Mesenchymal stem cells from CML patients were treated with imatinib (IM), and/or AG490, and/or E6201 for 3 days. BMP4-transcript expression from three samples is expressed in arbitrary units, and lines represent mean values \pm SEM, compared to untreated (NT). (C) TF1-BA and TF1-BAP cells treated or not with BMP4 (20 ng/mL for 24 hours) were analyzed for their content in P-Smad1/5/8 (left panel), or double positive P-Smad1/5/8-P-STAT3 (right panel). (D) TF1-BA cells were cultured in suspension or seeded on top of a HS27A layer and then treated for 6 days with IM $(1.5 \mu \mathrm{M})$ before viable cell number was analyzed. Scatter plot showing fold to input (day $6 /$ day 0 ), with dashed line representing no proliferation compared to input; $n=7$. (E) Experimental protocol for analysis of TKI persistence of leukemic cells promoted by stroma. TF1-BA cells were seeded on top of a HS27A layer for 3 or 6 days with imatinib (IM) $(1.5 \mu \mathrm{M})$, before adherent TF1-BA were harvested and further divided into suspended cells without IM or seeded on top of a HS27A layer with IM. (F) After 3 days, cell numbers were analyzed (left and middle) or colony forming cell (CFC) assays were performed (right). Scatter plots showing fold to input: Day 6 / Day 3 (left), Day 9 / Day 6 (middle), or CFC activity (right). Mann-Whitney test: * $P \leq 0.05$, ** $P \leq 0.01$. 
A
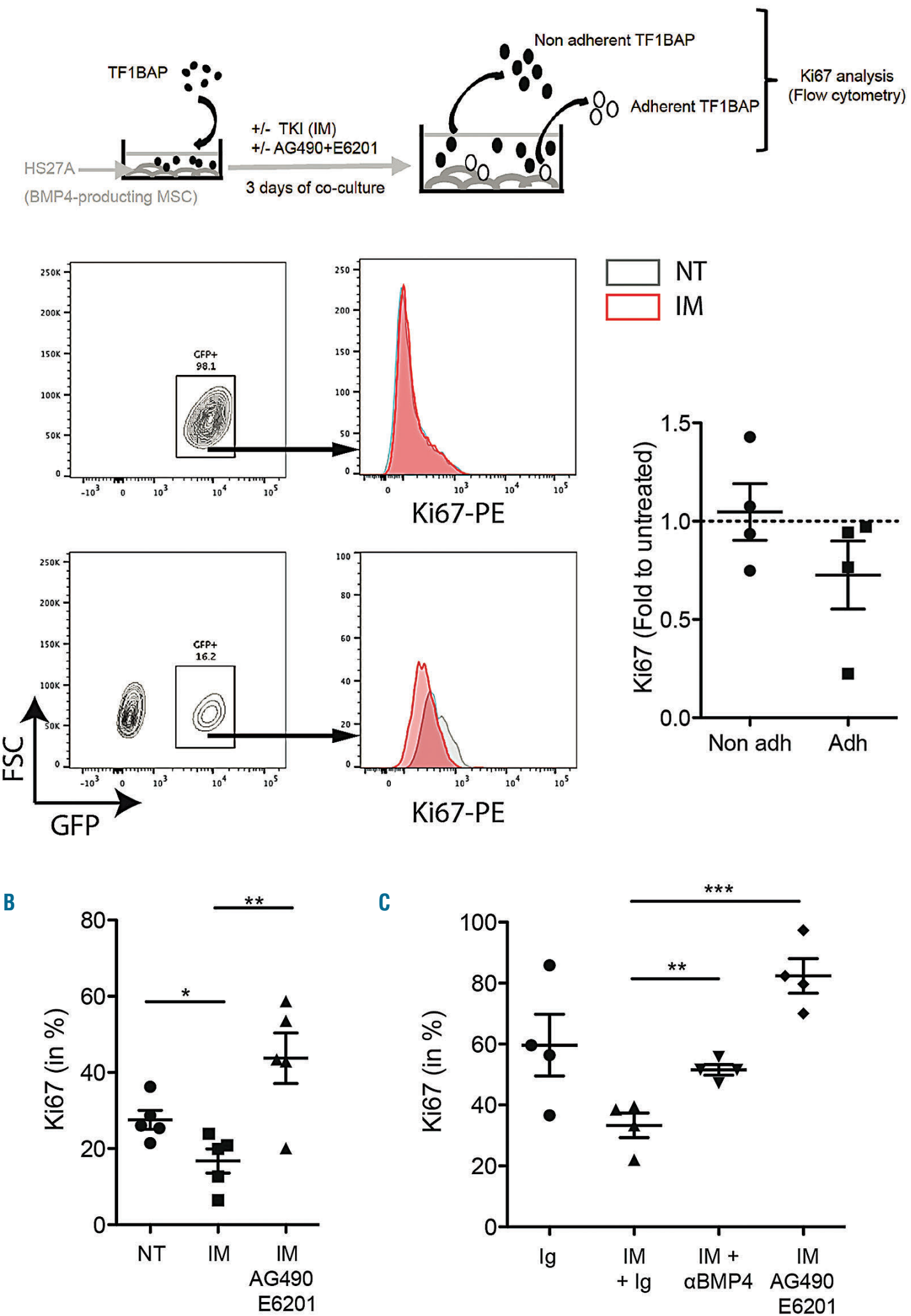

C

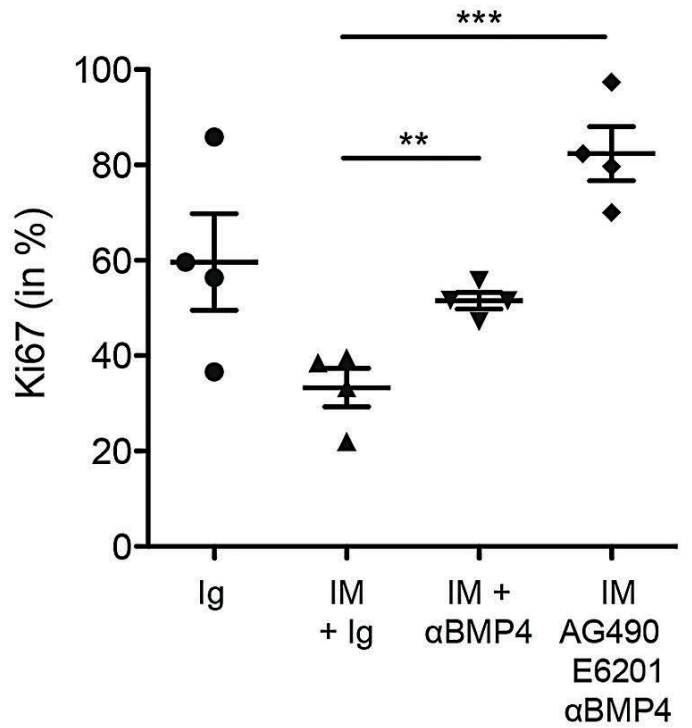

Figure 5. Targeting of chronic myeloid leukemia (CML) niche impacts the quiescence of persisting cells. (A-C) TF1-BAP cells were seeded on top of a HS27A layer and then treated for 3 days with imatinib (IM) $(2 \mu \mathrm{M})(\mathrm{A})$, with or without AG490 $(25 \mu \mathrm{M})+\mathrm{E} 6201(100 \mathrm{nM})(\mathrm{B}$ and $\mathrm{C})$, and addition of anti-BMP4 (C). Then TF1-BAP (GFP tagged) cells adherent and non-adherent fractions were harvested and analyzed separately for their content of Ki67-PE. Unpaired $t$-test: $* P \leq 0.05, * * P \leq 0.01$ $* * * P \leq 0.001$. NT: not treated. 

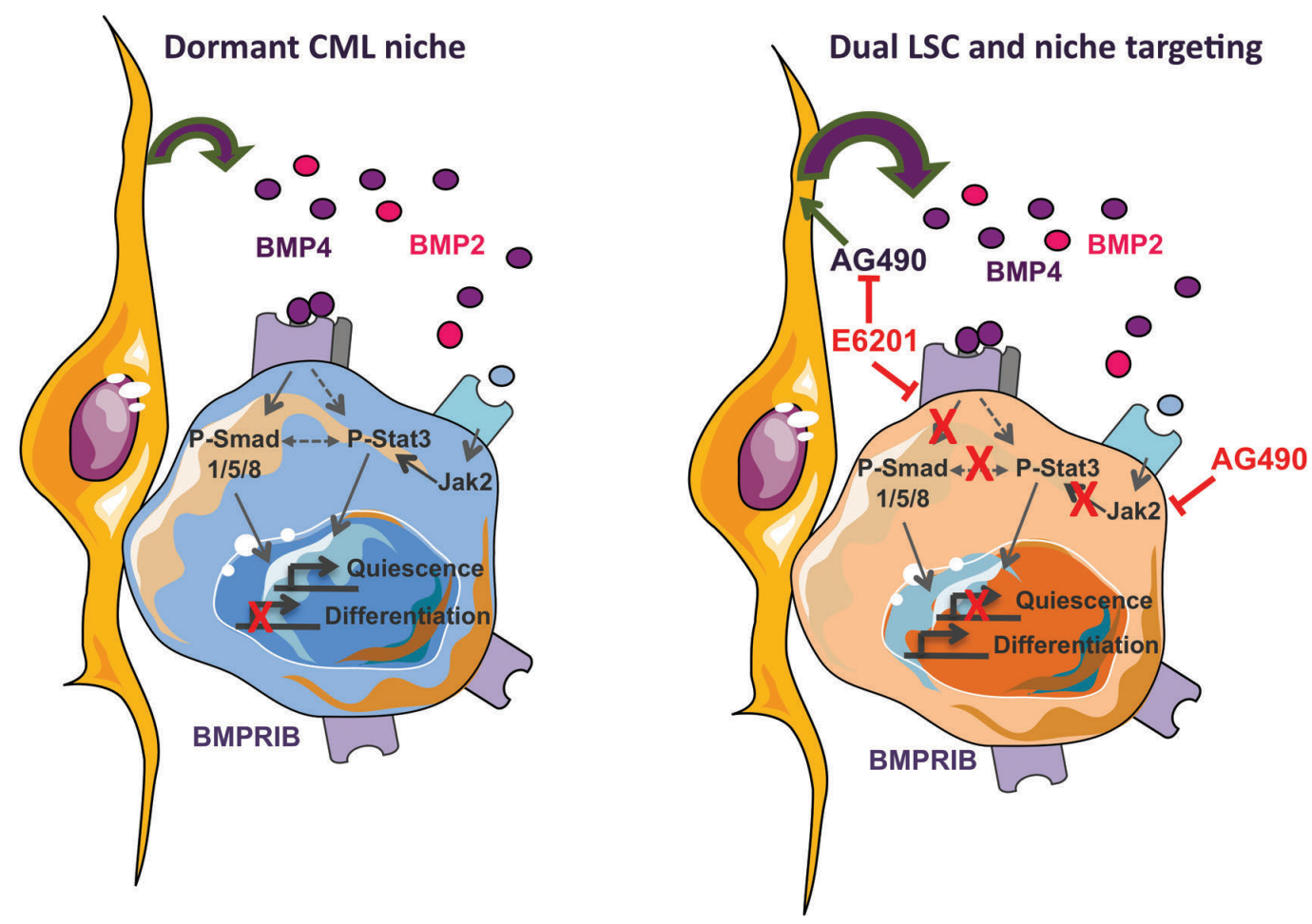

Figure 6. Proposed model to illustrate the regulation of quiescence stage of persisting chronic myeloid leukemia (CML) leukemic stem cells by their microenvironment. (Left) Leukemic stem cell dormancy of cells that interact with stromal cells of the niche is partly driven by BMP4 binding to its BMPR1B receptor over-expressed in those cells. This leads to phosphorylation of the Smad signaling (P-Smad 1/5/8) canonical pathway. It co-operates with the activation of Jak2/Stat3 signal through P-Stat3 induced directly by BMPR1B and/or by other cytokines binding to their receptors repressing proliferation while inducting quiescence genes expressions. (Right) Using a chemical inhibitor toward BMPR1B (E6201) in combination with a Jak2 inhibitor (AG490), targeting LSC could induce their proliferation and repress their quiescence. In addition, E6021 is preventing BMP4-induced production by stromal cells upon AG490 exposure, allowing a simultaneous dual targeting of the LSC and their leukemic protecting microenvironment.

and E6021 had no effect individually. BMP4 production induced by AG490 treatment was prevented by adding E6201 (Figure 4B).

In order to evaluate whether soluble BMP contribute to LSC persistence, we measured the impact of BMP4 treatment on Smad and Stat3 signaling. TF1-BA and TF1-BAP cells treated with exogenous BMP4 further increased their P-Smad1/5/8 and double positive P-Smad1/5/8-P-Stat3 levels (Figure 4C), while this treatment had no effect on cells displaying only P-Stat3 activation (Online Supplementary Figure S6D).

To evaluate the functional importance of BMP4 production by MSC, we then performed co-culture experiments of TF1-BA or TF1-BAP and HS27A cells (a model of BMP4producing MSC). ${ }^{22}$ We first observed that, despite the fact that TF1-BA cells in suspension were sensitive to IM, the addition of HS27A cells increased their persistence following 6 days of TKI treatment (Figure 4D). Drug withdrawal led TF1-BA cells to re-establishing a high level of proliferation after 3 (Figure 4E, left panel) or 6 (Figure 4E, right panel) days of co-culture with HS27A in the presence of TKI. In addition, release from the niche leads to clonogenic expansion in favor of TKI-treatment arrest (Figure 4F).

In order to evaluate the effect of BMP4 provided by the stroma on long-term persistent LSC, we separately analyzed adherent and non-adherent TF1-BAP cell fractions following 3 days of HS27A co-culture (Figure 5A). Flow cytometry analysis provided Ki67 expression (proliferation marker) of hematopoietic cells exclusively by gating GFP-expressing TF1-BAP cells. Figure 5A clearly shows a decrease in $\mathrm{Ki} 67^{+}$cells in adherent TKI-treated TF1-BAP cells, while the non-adherent fraction expressed similar Ki67 levels as untreated cells (normalized to 1). This effect on proliferation appeared to be dependent on the activation of BMP and Jak2 signaling, as it was reversed in the presence of both AG490 and E6201 (Figure 5B). The importance of BMP4 to mediate TKI-induced quiescence was confirmed by the absence of any significant effect when TF1-BAP cells were co-cultured with HS5 MSC (isolated from the BM of the same patient as HS27A) that express lower levels of BMP4 (Online Supplementary Figure $S 7 A$ ), on which TKI did not induce a decrease in $\mathrm{Ki}^{+}$ cells (Online Supplementary Figure S7B). Lastly, the addition of anti-BMP4 blocking antibody further demonstrates that the decrease in Ki67 following stroma co-culture under TKI treatment is impaired by inhibiting the BMP4 signal (Figure 5C), and can potentiate the effect of AG490 and E6201.

Overall, these data suggest that BMP4 controls Stat3 activation through Smad1/5/8 in persistent LSC to favor the maintenance of low cycling cells close to BMP4expressing stromal cells.

\section{Discussion}

In CML, the oncogene BCR-ABL alters numerous signaling pathways that control HSC, such as the BMP pathway that sustains the survival of $\mathrm{BMPR} 1 \mathrm{~B}^{+} \mathrm{LSC}$ and pro- 
genitors. ${ }^{22}$ Under continuous pressure of the BCR-ABLspecific TKI-treatment, LSC evolved and established a BMP4 autocrine loop, which contributes to treatment escape.$^{26}$ Furthermore, a sub-fraction of LSC survives in both TKI-sensitive or -resistant CML patients. ${ }^{7}$ Here, we revealed that, in the BM of CML patients in remission, residual LSC that displayed a quiescent molecular signature are also enriched in BMP signaling. Moreover, we show that a sub-fraction of quiescent cells presents a correlation between BMPR1B expression and the Stat3 pathway, but not with Stat5. Both BMPR1B ${ }^{26}$ and Stat $3^{43}$ have been implicated in CML LSC resistance/persistence to TKI treatment independently of the BCR-ABL oncogene. ${ }^{26,44}$ Targeting Jak2 and/or Stat3 alone or in combination with other pathways has been proposed to constitute an efficient way to target LSC and counteract their TKI resistance. ${ }^{45-47}$ Here, using primary cells and a novel model of $\mathrm{CD} 34^{+} \mathrm{CD} 38^{-}$TKI persistent LSC, we demonstrate that BMPR1B expression is linked to P-Smad1/5/8 and P-Stat 3 activation, and that concomitant targeting of these pathways impairs cell proliferation in suspension. This raised the hypothesis that the BMP pathway is responsible for the Stat3 activation in a small subset of persisting LSC. Indeed, we showed that BMP4 controls the concomitant activation of Smad1/5/8 and Stat3 in TKI persistent $\mathrm{CD} 34^{+}$cells, identifying the soluble factor produced by stromal cells and involved in the JAK/STAT3 activation as suggested by Kuepper et al. ${ }^{43}$ This is in accordance with results obtained in embryonic SC, showing that BMP controls Stat3 expression to sustain self-renewal and prevents cell differentiation through the induction of Id gene expression. ${ }^{48}$ At a molecular level, it is interesting to note that transcription factor binding site analysis revealed that Smad1 and Stat3 often co-occupy similar loci $^{49}$ and that Jak2 signaling regulates CML LSC.,45 Moreover, a direct link between the BMP and Jak/Stat pathway has been established in the neural system through the identification of a molecular complex between Smad1 and Stat3. ${ }^{50}$

We have previously demonstrated that BMP4 regulates human normal megakaryopoiesis, which is prevented in the presence of the Jak2 inhibitor, AG490, but not of MEK1/2 inhibitors. ${ }^{17}$ In addition, we have shown that TPO-induced megakaryopoiesis was partly mediated by the induction of a BMP4/BMPR1 autocrine loop. ${ }^{17}$ In the context of stem cell dormancy, TPO has been identified in an in vivo mouse model as a master cytokine controlling HSC quiescence in the BM niche through the Jak2 pathway. ${ }^{51}$ Moreover, BMP4 was shown to induce SC quiescence in different tissues such as the eyes, ${ }^{52}$ neural system, ${ }^{53-55}$ and skin. ${ }^{56}$ In keratinocytes, BMP4 induces SC dormancy by controlling DNA hypomethylation. ${ }^{56}$ In the neural system, BMP4 decreases cell proliferation through P27Kip1 regulation ${ }^{53}$ and prevents neural SC differentiation. ${ }^{55}$ Altogether, these observations suggest that BMP4 could directly control CML LSC quiescence through its modulation of the JAK/Stat3 pathway in a non-canonical signal, dependent upon BMPR1B kinase activity. We now provide evidence that residual $\mathrm{BM} \mathrm{BMPR} 1 \mathrm{~B}^{+} \mathrm{LSC}$ include (at least) two distinct subsets of leukemic persisting cells. In vitro data obtained with the TF1-BAP model of persisting immature $\mathrm{BCR}-\mathrm{ABL}^{+}$cells indicate that cell adhesion to stromal BMP4-producing MSC results in a drastically decreased proliferation in the presence of TKI, mediated by BMPR1B and Jak2 signaling. Conversely, non-adherent
BMPR1B+ TF1-BAP cells do not display a decrease in Ki67 upon TKI treatment. These data suggest that LSC could be maintained into a quiescent stage through a synergistic mechanism between the BMP signaling and adhesion to BM stromal cells. Indeed, in the drosophila model, it has been demonstrated that integrin signaling enhances BMP pathway activation through interactions with the BMP receptors independently of the FAK-signaling. ${ }^{57}$ In addition, the authors showed that integrin themselves are a target of BMP signaling. Moreover, extracellular matrix molecules such as integrin promote Stat3-dependent mitochondrial functions and cell survival through integrin-FAK signaling. ${ }^{58}$ Murine BMP4 regulates the expression of integrin alpha4 in HSC through a Smad-independent non-canonical signal. ${ }^{16}$ BMP4 induces HSC adhesion to stromal cells resulting in an increase in stem cell maintenance and expansion. ${ }^{17}$ Taken together, these data suggest that integrin could contribute to maintaining and amplifying the BMP4 signal to promote adherent LSC persistence. Since LSC of group A appeared to constitute a reservoir from which leukemic resistant or progressing cells could re-emerge, ${ }^{7}$ we hypothesized that these characteristics may enable the targeting of those persistent and quiescent LSC in their niche. Indeed, we then demonstrated that the combination of AG490-E6201 is optimal to prevent BMP4-induced MSC protection, reinforcing the importance of combining BMPR1B inhibition with Jak2 targeting.

Taken together, our results raise the hypothesis that close proximity of a subset of LSC to BMP4-producing MSC of the leukemic niche, increases the binding rate of BMP4 to its receptor BMPR1B to promote cell cycle exit in co-operation with integrin signaling and amplified by TKI exposure (Figure 6). This subpopulation of dormant LSC then re-enter the cell cycle upon TKI treatment cessation to slowly expand, progress, ${ }^{7}$ and mediate patient relapse after several months, as observed in $60 \%$ of the cases reported in the multicenter Stop Imatinib (STIM) clinical trial of treatment arrest. ${ }^{59}$

In conclusion, we identified BMP4/BMPR1B as a major bi-directional signal driving microenvironment-dependent LSC persistence alongside the Jak2-Stat3 pathway. Our data may prompt new therapeutic strategies to efficiently target LSC and their microenvironment, and prevent disease persistence during the treatment of CML and other leukemic malignancies.

\section{Disclosures \\ No conflicts of interest to disclose.}

\section{Contributions}

SJ, KA, ST, EG, KG and SL performed experiments and data analysis; TV developed the LSC persistent model; F-EN provided CML patients and donor samples and related clinical follow-up; AJM and ST designed protocols and data analysis for single cell RNA-Seq experiments; AG, SG-G and OG designed protocols and data analysis for Fluidigm RNA quantification; SL and VM-S designed the study, analyzed data and wrote the manuscript. All authors approved the final manuscript.

\section{Acknowledgments}

We thank P. Battiston (Cytometry Facility, CRCL, Lyon) for her technical assistance, and B. Manship for English editing. We thank Dr T. Myers from Spirita Oncology for providing us with E6201. 


\section{Funding}

Funding is acknowledged from LMC France patients' association and its president Mrs Mina Daban, "Fondation de France" 2014-0047501 and 2017-00076282/Fondation Ramona Ehrman Amador, "Association Laurette Fugain"
ALF2014-03, Ligue contre le Cancer (Haute Savoie, Loire, Puy de Dôme and Rhone), "Association ALTE-SMP", PLASCAN grants to VM-S, SL, and/or F-EN. PhD fellowship was obtained from "Ligue contre le cancer" (KA).

\section{References}

1. Kantariian HM, O'Brien S, Cortes JE, et al. Complete cytogenetic and molecular responses to interferon-alpha-based therapy for chronic myeloid leukemia are associated with excellent long-term prognosis. Cancer. 2003;97(4):1033-1041.

2. Simonsson B, Gedde-Dahl T, Markevarn B, et al. Combination of pegylated IFNalpha $2 b$ with imatinib increases molecular response rates in patients with low- or intermediate-risk chronic myeloid leukemia. Blood. 2011:118(12):3228-3235

3. Chu S, McDonald T, Lin A, et al. Persistence of leukemia stem cells in chronic myeloid leukemia patients in prolonged remission with imatinib treatment. Blood. 2011; 118(20):5565-5572

4. Chomel J-C, Bonnet M-L, Sorel N, et al Leukemic stem cell persistence in chronic myeloid leukemia patients with sustained undetectable molecular residual disease. Blood. 2011;118(13):3657-3660.

5. Rousselot P, Charbonnier A, Cony-Makhoul $\mathrm{P}$, et al. Loss of major molecular response as a trigger for restarting tyrosine kinase inhibitor therapy in patients with chronicphase chronic myeloid leukemia who have stopped imatinib after durable undetectable disease. J Clin Oncol. 2014;32(5):424-430.

6. Warfvinge R, Geironson L, Sommarin MNE, et al. Single-cell molecular analysis defines therapy response and immunophenotype of stem cell subpopulations in CML. Blood. 2017;129(17):2384-2394

7. Giustacchini A, Thongjuea S, Barkas N, et al. Single-cell transcriptomics uncovers distinct molecular signatures of stem cells in chronic myeloid leukemia. Nat Med. 2017; 23(6):692-702

8. Zhang B, Li L, Ho Y, et al. Heterogeneity of leukemia-initiating capacity of chronic myeloid leukemia stem cells. J Clin Invest. 2016;126(3):975-991

9. Graham SM, Jorgensen HG, Allan E, et al. Primitive, quiescent, Philadelphia-positive stem cells from patients with chronic myeloid leukemia are insensitive to STI571 in vitro. Blood. 2002;99(1):319-325

10. Hamilton A, Helgason GV, Schemionek M, et al. Chronic myeloid leukemia stem cells are not dependent on Bcr-Abl kinase activity for their survival. Blood. 2012;119(6):15011510.

11. Houshmand M, Simonetti G, Circosta P, et al. Chronic myeloid leukemia stem cells. Leukemia. 2019;33(7):1543-1556.

12. Agarwal P, Isringhausen $\mathrm{S}$, Li H, et al Mesenchymal niche-specific expression of Cxcl12 controls quiescence of treatmentresistant leukemia stem cells. Cell Stem Cell. 2019:24(5):769-784.e6.

13. Hogan BL. Bone morphogenetic proteins: multifunctional regulators of vertebrate development. Genes Dev. 1996;10(13):15801594.

14. Goldman DC, Bailey AS, Pfaffle DL, Masri AA, Christian JL, Fleming WH. BMP4 regulates the hematopoietic stem cell niche.
Blood. 2009:114(20):4393-4401.

15. Hutton JF, Rozenkov V, Khor FSL, D'Andrea RJ, Lewis ID. Bone morphogenetic protein 4 contributes to the maintenance of primitive cord blood hematopoietic progenitors in an ex vivo stroma-noncontact co-culture system. Stem Cells Dev. 2006;15(6):805-813.

16. Khurana S, Buckley S, Schouteden S, et al. A novel role of BMP4 in adult hematopoietic stem and progenitor cell homing via Smad independent regulation of integrin-alpha4 expression. Blood. 2013;121(5):781-790.

17. Jeanpierre S, Nicolini FE, Kaniewski B, et al. BMP4 regulation of human megakaryocytic differentiation is involved in thrombopoietin signaling. Blood. 2008;112(8):31543163.

18. Maguer-Satta V, Bartholin L, Jeanpierre S, et al. Regulation of human erythropoiesis by activin $\mathrm{A}, \mathrm{BMP} 2$, and $\mathrm{BMP} 4$, members of the TGFbeta family. Exp Cell Res. 2003;282(2): $110-120$.

19.Zylbersztejn F, Flores-Violante M, Voeltzel T, Nicolini FE, Lefort S, Maguer-Satta V. The BMP pathway: a unique tool to decode the origin and progression of leukemia. Exp Hematol. 2018;61:36-44.

20. Raymond A, Liu B, Liang $\mathrm{H}$, et al. A role for BMP-induced homeobox gene MIXL1 in acute myeloid leukemia and identification of type I BMP receptor as a potential target for therapy. Oncotarget. 2014;5(24):1267512693.

21. Voeltzel T, Flores-Violante M, Zylbersztejn $F$, et al. A new signaling cascade linking BMP4, BMPR1A, DeltaNp73 and NANOG impacts on stem-like human cell properties and patient outcome. Cell Death Dis. 2018;9(10):1011.

22. Laperrousaz B, Jeanpierre S, Sagorny K, et al. Primitive CML cell expansion relies on abnormal levels of BMPs provided by the niche and on BMPRIb overexpression. Blood. 2013;122(23):3767-3777.

23. Toofan P, Irvine D, Hopcroft L, Copland M, Wheadon $\mathrm{H}$. The role of the bone morphogenetic proteins in leukaemic stem cell persistence. Biochem Soc Trans. 2014; 42(4):809-815

24. Gerber JM, Gucwa JL, Esopi D, et al. Genome-wide comparison of the transcriptomes of highly enriched normal and chronic myeloid leukemia stem and progenitor cell populations. Oncotarget. 2013;4(5):715728.

25. Toofan P, Busch C, Morrison $\mathrm{H}$, et al. Chronic myeloid leukaemia cells require the bone morphogenic protein pathway for cell cycle progression and self-renewal. Cell Death Dis. 2018;9(9):927.

26. Grockowiak E, Laperrousaz B, Jeanpierre S, et al. Immature CML cells implement a BMP autocrine loop to escape TKI treatment. Blood. 2017;130(26):2860-2871

27. Cosset E, Hamdan G, Jeanpierre S, et al. Deregulation of TWIST-1 in the CD34+ compartment represents a novel prognostic factor in chronic myeloid leukemia. Blood. 2011;117(5):1673-1676.

28. Baccarani M, Deininger MW, Rosti G, et al. European LeukemiaNet recommendations for the management of chronic myeloid leukemia: 2013. Blood. 2013;122(6):872-884

29. Schallmoser K, Rohde E, Reinisch A, et al Rapid large-scale expansion of functional mesenchymal stem cells from unmanipulated bone marrow without animal serum. Tissue Eng Part C Methods. 2008;14(3):185196.

30. Sheng Y, Ji Z, Zhao H, et al. Downregulation of the histone methyltransferase SETD2 promotes imatinib resistance in chronic myeloid leukaemia cells. Cell Prolif. 2019; 52(4):e12611.

31. Richard A, Boullu L, Herbach U, et al. Singlecell-based analysis highlights a surge in cellto-cell molecular variability preceding irreversible commitment in a differentiation process. PLoS Biol. 2016;14(12):e1002585.

32. Byron SA, Loch DC, Wellens CL, et al. Sensitivity to the MEK inhibitor E6201 in melanoma cells is associated with mutant BRAF and wildtype PTEN status. Mol Cancer. 2012;11:75

33. Zhang W, Borthakur G, Gao C, et al. The dual MEK/FLT3 inhibitor E6201 exerts cytotoxic activity against acute myeloid leukemia cells harboring resistance-conferring FLT3 mutations. Cancer Res. 2016;76(6):1528-1537.

34. Babiker HM, Byron SA, Hendricks WPD, et al. E6201, an intravenous MEK1 inhibitor, achieves an exceptional response in BRAF V600E-mutated metastatic malignant melanoma with brain metastases. Invest New Drugs. 2019;37(4):636-645.

35. Kitamura T, Tange T, Terasawa $T$, et al. Establishment and characterization of a unique human cell line that proliferates dependently on GM-CSF, IL-3, or erythropoietin. J Cell Physiol. 1989;140(2):323-334.

36. Abraham SA, Hopcroft LE, Carrick E, et al. Dual targeting of p53 and c-MYC selectively eliminates leukaemic stem cells. Nature. 2016:534(7607):341-346.

37. Das B, Pal B, Bhuyan R, et al. MYC regulates the HIF2alpha stemness pathway via Nanog and Sox2 to maintain self-renewal in cancer stem cells versus non-stem cancer cells. Cancer Res. 2019;79(16):4015-4025.

38. Pellicano F, Scott MT, Helgason GV, et al. The antiproliferative activity of kinase inhibitors in chronic myeloid leukemia cells is mediated by FOXO transcription factors. Stem Cells. 2014;32(9):2324-2337.

39. Chen Y, Hu Y, Zhang H, Peng C, Li S. Loss of the Alox5 gene impairs leukemia stem cells and prevents chronic myeloid leukemia. Nat Genet. 2009:41(7):783-792.

40. Nievergall E, Ramshaw HS, Yong AS, et al. Monoclonal antibody targeting of IL-3 receptor alpha with CSL362 effectively depletes CML progenitor and stem cells. Blood. 2014;123(8):1218-1228

41. Carter BZ, Mak PY, Mu H, et al. Combined targeting of BCL-2 and BCR-ABL tyrosine kinase eradicates chronic myeloid leukemia stem cells. Sci Transl Med. 2016; 8(355):355ra117.

42. Hamilton A, Helgason GV, Schemionek M, et al. Chronic myeloid leukemia stem cells are not dependent on Bcr-Abl kinase activity 
for their survival. Blood. 2012;119(6):15011510.

43. Kuepper MK, Butow M, Herrmann O, et al. Stem cell persistence in CML is mediated by extrinsically activated JAK1-STAT3 signaling. Leukemia. 2019;33(8):1964-1977.

44. Eiring AM, Kraft IL, Page BD, O'Hare T, Gunning PT, Deininger MW. STAT3 as a mediator of BCR-ABL1-independent resistance in chronic myeloid leukemia. Leuk Suppl. 2014;3(Suppl 1):S5-S6.

45. Gallipoli P, Cook A, Rhodes S, et al. JAK2/STAT5 inhibition by nilotinib with ruxolitinib contributes to the elimination of CML CD34+ cells in vitro and in vivo. Blood. 2014;124(9):1492-1501.

46. Gleixner KV, Schneeweiss M, Eisenwort G, et al. Combined targeting of STAT3 and STAT5: a novel approach to overcome drug resistance in chronic myeloid leukemia. Haematologica. 2017;102(9):1519-1529.

47. Chakraborty SN, Leng X, Perazzona B, Sun $\mathrm{X}$, Lin YH, Arlinghaus RB. Combination of JAK2 and HSP90 inhibitors: an effective therapeutic option in drug-resistant chronic myeloid leukemia. Genes Cancer. 2016;7(56):201-208.

48. Ying OL, Nichols J, Chambers I, Smith A. $\mathrm{BMP}$ induction of Id proteins suppresses dif- ferentiation and sustains embryonic stem cell self-renewal in collaboration with STAT3. Cell. 2003;115(3):281-292.

49. Chen X, Xu H, Yuan P, et al. Integration of external signaling pathways with the core transcriptional network in embryonic stem cells. Cell. 2008;133(6):1106-1117.

50. Nakashima K, Yanagisawa M, Arakawa H, et al. Synergistic signaling in fetal brain by STAT3-Smad1 complex bridged by p300. Science. 1999;284(5413):479-482.

51. Bersenev A, Wu C, Balcerek J, Tong W. Lnk controls mouse hematopoietic stem cell selfrenewal and quiescence through direct interactions with JAK2. J Clin Invest. 2008; 118(8):2832-2844

52. Balenci L, Wonders C, Coles BL, Clarke L, van der Kooy D. Bone morphogenetic proteins and secreted frizzled related protein 2 maintain the quiescence of adult mammalian retinal stem cells. Stem Cells. 2013;31(10):2218-2230.

53. Andreu Z, Khan MA, Gonzalez-Gomez P, et al. The cyclin-dependent kinase inhibitor p27 kip1 regulates radial stem cell quiescence and neurogenesis in the adult hippocampus. Stem Cells. 2015;33(1):219-229.

54. Han X, Yu L, Chen O, et al. BMP4/LIF or RA/Forskolin suppresses the proliferation of neural stem cells derived from adult monkey brain. Stem Cells Int. 2017;2017:7012405.

55. Bond AM, Peng CY, Meyers EA, McGuire T, Ewaleifoh O, Kessler JA. BMP signaling regulates the tempo of adult hippocampal progenitor maturation at multiple stages of the lineage. Stem Cells. 2014;32(8):2201-2214.

56. Lee J, Kang S, Lilja KC, et al. Signalling couples hair follicle stem cell quiescence with reduced histone H3 K4/K9/K27me3 for proper tissue homeostasis. Nat Commun. 2016;7:11278.

57. Sawala A, Scarcia M, Sutcliffe C, Wilcockson SG, Ashe HL. Peak BMP responses in the drosophila embryo are dependent on the activation of integrin signaling. Cell Rep. 2015;12(10):1584-1593.

58. Visavadiya NP, Keasey MP, Razskazovskiy $\mathrm{V}$, et al. Integrin-FAK signaling rapidly and potently promotes mitochondrial function through STAT3. Cell Commun Signal. 2016; 14(1):32.

59. Mahon FX, Rea D, Guilhot J, et al Discontinuation of imatinib in patients with chronic myeloid leukaemia who have maintained complete molecular remission for at least 2 years: the prospective, multicentre Stop Imatinib (STIM) trial. Lancet Oncol. 2010;11(11):1029-1035. 